\title{
A REVIEW ON SYNTHESIS OF SILVEFR NANOPARTICLES
}

\section{PATEL $\mathrm{H}^{1}$, ANDHARE $\mathrm{P}^{2}$, MARCHAWALA $\mathrm{F}^{2}$, BHATTACHARYA $\mathrm{I}^{2}$ AND UPADHYAY $\mathbf{D}^{2 *}$}

1: Student, M. Sc. Microbiology, Parul Institute of Applied Sciences, Parul University, Post Limda,

$$
\text { Waghodia, Gujarat, } 391760
$$

2: Assistant Professor, Parul Institute of Applied Sciences, Parul University, Post Limda, Waghodia, Gujarat, 391760

*Corresponding Author: Dr. Dhwani Upadhyay; E Mail:dhwani.updhyay82123@paruluniversity.ac.in;

Tel: +919558021474

Received 22 ${ }^{\text {nd }}$ Jan. 2021; Revised 24 ${ }^{\text {th }}$ Feb. 2021; Accepted $25^{\text {th }}$ March 2021; Available online $1^{\text {st }}$ April 2021

\section{https://doi.org/10.31032/IJBPAS/2021/10.4.1034}

\begin{abstract}
Silver nanoparticles (NPs) are loosely utilized in totally different fields of science. Their exclusive things, which may remain combined in biosensor materials, compound fibers, beautifying product, antimicrobial uses, leading materials and electric elements, build them an awfully vital subject to be studied by chemistry, biology, healthcare, electronic and alternative connected branches. These exclusive properties rely on size and form of the silver nanoparticles. For the synthesis of silver nanoparticles, various preparation techniques are used, such as lepton irradiation, optical maser ablation, chemical reduction, biological artificial strategies, chemical research strategies, and microwave process. The objectives of this report is to create silver nanoparticles from a variety of plant sources. A thorough analysis of the reduction of silver ions to silver nanoparticles mediated by plant leaves extract revealed that the process was simple and fast. Characterization of the synthesized nanoparticles can be performed through UV spectroscopic analysis, Fourier remodel Infra-Red spectroscopic analysis analysis, diffraction analysis, Scanning microscopy and High Resolution Transmission microscopy were relatively analysed for the absorbance, stabilization of bonds, particle sizes in terms of $\mathrm{nm}$ and therefore the particle shapes contributive configuration severally.
\end{abstract}

Keywords: Silver nanoparticles, physical methods, chemical methods, green synthesis 


\section{INTRODUCTION}

Nanotechnology is also a main field of recent analysis managing vogue, synthesis, and manipulation of particle structures reaching from a lot of or less 1-100nm [1]. Because of their unusual properties, they've been used in a variety of uses, such as prescription agents, in manufacturing, domestic, and healthcare-related products, in consumer products, medical equipment coatings, optical sensors, and cosmetics, in the pharmaceutical and food industries, in medicine, medical research, drug distribution, and as malignant tumour agents, and have eventually inflated the tumor-killing effects of malignant tumor medication. Because of their surface-to-volume ratio, nanosized argentiferous particles unit of measurement exclusive and can greatly modify physical, chemical, and biological properties; thus, these nanoparticles are defeated for several resolves [2, 3]. Following synthesis, detailed component classification is expected, as a particle's chemical science properties can have a significant impact on its biological properties. To resolve the safety issue of using the full potential of every Nano substance in the resolution of human safety, in Nano medicines, or in the health-care industry, it's necessary to first describe the different nanoparticles [4]. The characteristic feature of nanomaterials, for instance size, shape, size circulation, extent, shape, solubility, aggregation, etc. wish to be valued earlier activity poisonousness or biocompatibility [5]. The biological activity of AgNPs is influenced by a number of factors, including surface chemistry, size, size distribution, shape, particle morphology, particle composition, coating/capping, agglomeration, and dissolution rate, particle reactivity in resolution, particle unharness potency, and cell kind, and hence the reasonably reducing agents used in AgNP synthesis are a significant issue [6].

\section{SYNTHESIS OF AgNPS}

\section{Physical methods}

Vaporization-reduction and laser ablation are the two most effective physical approaches [7]. Physical synthesis techniques, in contrast to chemical processes, are distinguished by the absence of solvent contamination in armed thin films and the regularity of NP delivery. Tube furnace syntheses of silver NPs by atmospheric force have a number of drawbacks, including energy consumption, sluggish synthesis, and a high concentration requirement.

Laser ablation of metallic majority resources can be synthesized AgNPs in solution. Depends upon several elements, with the wavelength of the laser disturbing the metallic 
target, the period of the laser pulses, the ablation time amount and the able liquid medium, with or without the existence of surfactants, and the laser power are few of the factors which define the ablation efficiency and the features of synthesized nano-silver elements.Physical synthesis processes, as opposed to chemical approaches, are characterised by the absence of solvent infection in armed thin films and the homogeneity of NP circulation. Tube furnace syntheses of silver NPs at atmospheric pressure have a number of drawbacks, including high energy consumption, slow synthesis, and a high concentration need.Laser ablation of metallic majority resources can be synthesized AgNPs in solution.The ablation performance and features of synthesised Nano silver particles are determined by a number of factors, including the wavelength of the laser interjecting the metallic target, the duration of the laser pulses, the ablation time amount, the effective liquid medium, with or without surfactants, and the laser strength [8].

\section{Chemical method}

Chemical reduction, which uses organic and inorganic reducing agents, is the most effective method for producing AgNPs. Because of the external of a metal with free of charge electrons in the conduction band and a positively charged nucleus, this is done by continuing into a specific process to produce a coloured silver solution. Prior to the synthesis of AgNPs, the formulation of long-lasting silver bunches is formed and verified [9]. Generally, one-pot technique of reduction of $\mathrm{AgNO}_{3}$ spending dissimilar reducing agents for example sodium citrate, ascorbate [10].

Because of their unique properties, such as ultralow interfacial stress, large interfacial surface, thermodynamic constancy, and the capacity to solubilize immiscible liquids, micro emulsions have a variety of applications in the chemical and biological fields. The Micro emulsion technique declares to be one of the versatile preparation methods that allows for the organisation of element properties such as element size control mechanisms, structure, morphology, homogeneity, and surface area [11].

A microwave synthesis involves the reduction of silver nanoparticles with variable rate microwave radiation in opposition to the conventional heating method [12]. The method gives up an extra quickly reaction and gives a higher concentration of silver nanoparticles through the equal heat and contact [7].

\section{Biological method}

In recent times, the biological method for synthesizing AgNPs is being progressively 
measured. This method is a green technology targeted at minimalizing the harmful environmental impression. It had been known that the synthesis of AgNPs consuming the chemical method needs three main elements: a silver salt, a reducing agent, and a stabilizer or capping agent. In the biological method, the dropping agent and the stabilizer are changed using molecules gained from living organisms for example plants, bacteria, fungi, yeast, and algae; their specifics are conversed in the following sections [13].

\section{CHARACTERIZATION OF SILVER NANOPARTICLES}

Characterization of nanoparticles is important to grasp and supervisor nanoparticles synthesis and applications. Characterization is performed employing a modification of dissimilar strategies for instance transmission and scanning lepton microscopy(TEM, SEM), atomic force research (AFM), dynamic light-weight scattering (DLS), X-ray negatron chemical analysis (XPS),powder X-ray diffractometric (XRD), Fourier remodel infrared chemical analysis (FTIR), and UV-Vis chemical analysis. These strategies ar future for determination of dissimilar parameters as an example particle size, shape, crystallinity, pattern dimensions, pore size and extent. What is more, orientation, interval and dispersion of nanoparticles and nanotubes in nanocomposite materials may be determined by these techniques [14]. For instance, the morphology and part size may be determined through TEM, SEM and AFM. The advantage of AFM over ancient microscopes for instance SEM and TEM is that AFM measures three-dimensional photos so part height and volume are often calculated. What is more, dynamic light-weight scattering is planned for determination of particles size circulation. What is more, X-ray diffraction is meant for the determination of crystallinity, whereas $\mathrm{UV}-\mathrm{Vis}$ chemical analysis is employed to substantiate sample formation through showing the plasmon resonance [14].

\section{BIOLOGYCAL APPLICATION OF AgNPs}

\section{Antibacterial Activity of AgNPs}

The Ag-NPs have been shown to be an active biocide against a broad-spectrum bacteria that includes both Gram-negative and Gram-positive bacteria, as well as some highly pathogenic bacterial strains. This article summarises the antibacterial activities of Ag-NPs as reported in current journals. Surprisingly, together with the accretion of Ag-NPs in the bacterial membrane, the bacterial cells were damaged and destroyed. Mormons have also investigated the antibacterial activities of Ag-NPs in the 1-100 
nm range using a number of Gram-negative bacteria [15]. Additionally, Kim et al [16] have used a model of mutually Gram-negative (E. coli) and Gram-positive (S. aureus) bacteria to examine the antibacterial activities of Ag-NPs. Their research revealed that E. coli is resistant to Ag-NPs at low concentrations ( $3.3 \mathrm{nM})$, which is ten times lower than the smallest inhibitory dose for S. aureus (33nM).Shrivastava et al [17] identified the solid antibacterial power of novel Ag-NPs in the $10-15 \mathrm{~nm}$ range with enhanced immovability in the presence of a few non-resistant and drug-resistant bacteria strains. It was decided that the antibacterial consequence is dose-dependent and is extra noticeable against Gram-negative than Gram-positive bacteria; it was too self-regulating of acquisition of resistance by the bacteria beside antibiotics [15]. In a current review, Jones and Hoek condensed three common antibacterial mechanisms of Ag-NPs as follows: (i) uptake of free silver ions monitored by interruption of ATP production and DNA replication, (ii) Ag-NPs and silver ion group of reactive oxygen species (ROS) and (iii) Ag-NPs' straight destruction to cell membranes [18]. Obviously, with detected brilliant antibacterial properties, Ag-NPs have been advised as effective broad-spectrum biocides beside a range of drug-resistant bacteria, and a possible candidate for use in pharmaceutical and medical products acceptable to prevent the transmission of drug-resistant pathogens in dissimilar clinical environments [19].

\section{Antifungal Activity of AgNPs}

Fungal infections are most frequent in immunocompromised patients, and treating fungi-mediated diseases is a time-consuming process due to the small number of antifungal medications currently available [20]. Hence, there is an unavoidable and vital essential to progress antifungal agents, which should be biocompatible, non-toxic, and environmentally friendly. At this time, AgNPs show a significant role as anti-fungal agents in contradiction of many diseases affected by fungi. With concentrations of $1-7 \mathrm{~g} / \mathrm{ml}$, Nano-Ag demonstrated potent anti-fungal action against clinical isolates and ATCC strains of Trichophyton mentagrophytes and Candida species [21]. In a sodalime bottle, an inert matrix containing AgNPs with an average size of $20 \mathrm{~nm}$ was developed, which showed improved biocidal behaviour. Monodisperse Nano-Ag sepiolite fibers displayed important antifungal activity against Issatchenkia orientalis. AgNPs showed worthy antifungal activity against Aspergillus niger and a MIC of $25 \mu \mathrm{g} / \mathrm{mL}$ against Candida albicans. Biologically 
-synthesized AgNPs obtainable enhanced antifungal activity with fluconazole beside Phoma glomerata, Phomaherbarum, Fusarium semitectum, Trichoderma sp., andCandida albicans AgNPs steadied by sodium dodecyl sulphate presented improved antifungal activity beside Candida activity in contradiction of Candida albicans linked to predictable antifungal agents Candida albicans equalled to conventional antifungal agents. The size-dependent antifungal activities of dissimilar AgNPs were completed against mature Candida albicans and Candida glabrata biofilms. Biologically synthesized AgNPs showed antifungal activity against some phytopathogenic fungi, counting Alternaria alternata, Sclerotinia sclerotiorum, Macrophomina phaseolina, Rhizoctonia solani, Botrytis cinerea, and Curvularia lunata at the concentration of 15 mg [4].

\section{Antiviral Activity of AgNPs}

In latest days, there has been an increase in the number of confirmed cases of emerging and re-emerging infectious diseases caused by viruses like SARS-Cov, influenza A/H5N1, influenza A/H1N1, Dengue virus, HIV, HBV, and novel encephalitis viruses, among others. This viral infections are likely to develop into highly infectious diseases, posing a public health risk [22]. As the initial report,
Elechiguerra et al., have examined the collaboration among Ag-NPs and HIV-1. It was informed that Ag-NPs experience a size-dependentinteraction, with NPs completely in the variety of $1-10$ nminvolved to the virus. It was also suggested that Ag-NPs interact with the HIV-1 virus by binding to the exposed sulphur-bearing remains of the gp120 glycoprotein knobs, preventing the virus from binding to the host cell. In additional study, $\mathrm{Lu}$ et al explored the effects of Ag-NPs of different sizes $(10,50$ and $800 \mathrm{~nm})$ on thehepatitis $B$ virus (HBV), and expending a HepAD38 cell line asinfection model. Their study presented that only Ag-NPs couldconstrain production of HBV RNA and extracellular virionsin vitro. Moreover, Sun et al., have developed Ag-NPs conjugated with (N-vinyl-2-pyrrolidone) (PVP), recombinant respiratory syncytial virus (RSV) fusion (F) protein, andbovine serum albumin (BSA) acceptable to study the inhibitionof RSV infection in HEp-2 cell culture. The achievedresults exposed that PVP-coated silver nanoparticles, whichpresented small toxicity to cells at small concentrations, inhibitedRSV infection by $44 \%$, a significant reduction equalled toextra controls. New recently, Xiang et al examined the inhibitory things of Ag-NPs on H1N1 influenza $A$ virusin vitro. Theirstudy exposed that Ag-NPs 
have efficient inhibitory activityon H1N1 influenza a virus, which can quickly prevent H1N1 influenza a virus hemagglutination of chicken RBCs. Furthermore, Ag-NPs could also decrease HIN1 influenza a virus encouraged apoptosis to MDCK cells.Even so, the journalists recommended simplifying how Ag-NPs inhibit H1N1 influenza virus infection as well as the use of Ag-NPs as an active anti-influenza medicine [15].

\section{CONCLUSION}

AgNPs have been disapprovingly analysed from the standpoints of analysis dynamics, global use, synthesis, properties, and potential threats in this article. Generally, there are a unit 3 ways for synthesizing AgNPs, namely physical, chemical, and biological. The physical approach has many drawbacks, like giant area requirement, high energy consumption, and very long time consumption to realize thermal stability. The chemical approach provides a simple thanks to manufacture AgNPs although the toxicity of their by-products may be a primary concern. Inexperienced synthesis of AgNPs is turning into a lot of popular since it's environmentally friendly and low-cost. The present review showed that AgNPs have fascinating physical, chemical, biological, optical, thermal, electrical, and chemical action properties. These properties have the potential to be exploited in many applications. In the other hand, it's important to remember that AgNPs are poisonous, and should be considered when they're used in client products. The versatility of silver nanoparticle artificial methods and the ease of which silver NPs can be incorporated into a variety of media has prompted researchers to investigate the mechanistic implications of medicine, as well as the antiviral and antifungal effects of such NPs.Shape, size and size distribution of silver NPs are often controlled by adjusting the reaction conditions like reducer, stabilizer or using totally different artificial ways. Therefore, it's necessary to elucidate the results of reaction conditions on morphology and size of NPs.

\section{ACKNOWLEDGEMENT}

It's our privilege and honour to express our sincerest gratitude to the Parul University, Vadodara, Gujarat for providing me all the necessary support and facilities including state of the art infrastructural facilities with advanced technological scientific laboratories and everything else that was required to carry out this work.

\section{REFERENCES}

[1] Iravani, S., Korbekandi, et al. Synthesis of silver nanoparticles: chemical, physical and biological 
methods. Research in pharmaceutical sciences.Nov-Dec2014; 9(6): 385.

[2] Li, L.; Hu, J.; et al. A.P. Band gap variation of size- and shape-controlled colloidal CdSe quantum rods. Nano Lett. July2001; 1(7): 349-351.

[3] Sharma, V.K.; Yngard, R.A.; et al. Silver nanoparticles: Green synthesis and their antimicrobial activities. Adv. Colloid Interface.Jan2009; 145(1-2): 83-96.

[4] Zhang, X. F., et al. S. Silver nanoparticles: synthesis, characterization, properties, applications, and therapeutic approaches. International journal of molecular sciences. Sep 2016; 17(9): 1534.

[5] Murdock, R.C.; Braydich-Stolle, L.; et al. Characterization of nanomaterial dispersion in solution prior to in vitro exposure using dynamic light scattering technique. Toxicol. Sci.Sep 2008; 101(2): 239-253.

[6] Carlson, C.; Hussain, S.M.; et al. Unique cellular interaction of silver nanoparticles: Size-dependent generation of reactive oxygen species. J. Phys.Chem. B .Oct2008; 112(43): 13608-13619.

[7] Beyene, H. D., et al. Synthesis paradigm and applications of silver nanoparticles (AgNPs), a review. Sustainable materials and technologies.Sep2017; 13:18-23.

[8] N. Tarasenko, A. Butsen, et al. Synthesis of nanosized particles during laser ablation of gold in water, Appl. Surf. Sci. April 2006; 252(13): 4439-4444.

[9] J.I. Hussain, S. Kumar, et al. Silver nanoparticles: preparation, characterization, and kinetics, Adv. Mater. Lett. Mar 2011; 2(3): 188-194.

[10] M.J. Firdhouse, P. Lalitha end. Biosynthesis of silver nanoparticles and its applications, J. Nanotechnol. Oct 2015; 2015.

[11] F.S. Al-Mubaddel, S. Haider, et al. Engineered nanostructures: a review of their synthesis, characterization, and toxic hazard considerations, Arab. J. Chem. Feb 2012; 10:376-88.

[12] R. Veerasamy, T.Z. Xin, et al. Biosynthesis of silver nanoparticles using mangosteen leaf extract and evaluation of their antimicrobial activities, J. Saudi Chem. Soc. Apr 2012; 15(2): 113-120.

[13] Syafiuddin, A., et al. A review of silver nanoparticles: research trends, global consumption, synthesis, properties, and future 
challenges. Journal of the Chinese Chemical Society. Jul 2017; 64(7): 732-756.

[14] About El-Nour, K. M., Eftaiha, et al. Synthesis and applications of silver nanoparticles. Arabian journal of chemistry. Jul 2010; 3(3):135-140.

[15] Tran, Q. H., et al. Silver nanoparticles: synthesis, properties, toxicology, applications and perspectives. Advances in Natural Sciences: Nanoscience and Nanotechnology.May 2013; 4(3):033001.

[16] Kim J $\mathrm{S}$ et al. Nanomed. Nanotechnol. Mar 2007; 3(1): 95-101.

[17] Shrivastava S, Bera $\mathrm{T}$, et al. Nanotechnology. May 2007; 18(22): 2251031.

[18] Jones C M and Hoek E M V J end. Nanopart. Res. Jun 2010, 12(5): 1531-51.

[19] Lara H H, Garza-trevino E N, et al. Nanobiotechnol. Dec 2011; 9(1): $1-8$.

[20] Kim, K.J.; Sung, W.S.; et al. Antifungal effect of silver nanoparticles on dermatophytes. J. Microbiol. Biotechnol. Aug 2008; 18(8): 1482-1484.
[21] Esteban-Tejeda, L.; Malpartida, F.; et al. The antibacterial and antifungal activity of a soda-lime glass containing silver nanoparticles. Nanotechnology. Feb 2009; 20(8):085103.

[22] Coker R J, Hunter B M, et al. Emerging infectious diseases in Southeast Asia: regional challenges to control. The Lancet. Feb 2011; 377(9765): 599-609. 